\title{
Identification of genes and miRNA associated with idiopathic recurrent pregnancy loss: an exploratory data mining study
}

Wael Bahia ${ }^{1,2}$, Ismael Soltani ${ }^{3}$, Anouar Abidi ${ }^{4}$, Anis Haddad ${ }^{5}$, Salima Ferchichi ${ }^{1}$, Samia Menif ${ }^{3}$ and Wassim Y. Almawi ${ }^{6,7^{*}}$

\begin{abstract}
Background: Recurrent pregnancy loss (RPL) is a significant adverse pregnancy complication, with an incompletely understood pathology. While many entities were proposed to elucidate the pathogenic basis of RPL, only few were significant enough to warrant investigation in all affected couples.. The aim of this study was to provide novel insights into the biological characteristics and related pathways of differentially expressed miRNA (DEMs) and genes (DEGs), in RPL, and construct a molecular miRNAs-mRNAs network.

Methods: miRNAs and gene expression data were collected, and a number of DEMs and (DEGs) were obtained, and regulatory co-expression network were constructed. Function and enrichment analyses of DEMs were conducted using DIANA-miRPath. DEGs were screened, and were used in generation of protein-protein interaction (PPI) network, using STRING online database. Modularity analysis, and pathway identification operations were used in identifying graph clusters and associated pathways. DEGs were also used for further gene ontology (GO) analysis, followed by analysis of KEGG pathway.

Results: A total of 34 DEMs were identified, and were found to be highly enriched in TGF- $\beta$ signaling pathway, Fatty acid metabolism and TNF signaling pathway. Hub miRNAs were selected and were found to be involved in several functional pathways including progesterone-mediated oocyte maturation and Thyroid hormone signaling pathway. Five dysregulated feedback loops involving miRNA and TFs were identified and characterized. Most notably, PPI network analysis identified hub-bottleneck protein panel. These appear to offer potential candidate biomarker pattern for RPL diagnosis and treatment.
\end{abstract}

Conclusions: The present study provides novel insights into the molecular mechanisms underlying RPL.

Keywords: Bioinformatics analysis, Gene expression, microRNA, Recurrent pregnancy loss

* Correspondence: wassim.almawi@outlook.com

${ }^{6}$ Faculty of Sciences, El Manar University, Tunis, Tunisia

${ }^{7}$ College of Health Sciences, Abu Dhabi University, Abu Dhabi, United Arab Emirates

Full list of author information is available at the end of the article

\section{Background}

Recurrent pregnancy loss (RPL), defined as two or more consecutive pregnancy losses before the 20th week of gestation, is a significant pregnancy complication, affecting $2-5 \%$ of pregnancies [1]. While its exact etiology remains poorly understood [2] several risk factors were shown to influence the risk of RPL These include

(c) The Author(s). 2020 Open Access This article is licensed under a Creative Commons Attribution 4.0 International License, which permits use, sharing, adaptation, distribution and reproduction in any medium or format, as long as you give appropriate credit to the original author(s) and the source, provide a link to the Creative Commons licence, and indicate if changes were made. The images or other third party material in this article are included in the article's Creative Commons licence, unless indicated otherwise in a credit line to the material. If material is not included in the article's Creative Commons licence and your intended use is not permitted by statutory regulation or exceeds the permitted use, you will need to obtain permission directly from the copyright holder. To view a copy of this licence, visit http://creativecommons.org/licenses/by/4.0/ The Creative Commons Public Domain Dedication waiver (http://creativecommons.org/publicdomain/zero/1.0/) applies to the data made available in this article, unless otherwise stated in a credit line to the data. 
endocrine, immunologic, infectious, and genetic factors [3]. Whereas increased RPL susceptibility was linked with carriage of select gene variants, few of these at-risk variants was confirmed to contribute to RPL pathogenesis.

MicroRNAs (miRNA) are short (22-23 nucleotides) non-coding RNA, which regulate post-transcriptional activities [4], reportedly regulating $\sim 60 \%$ of all proteincoding human genes, and are involved in diverse physiological processes and pathological states [5]. Their activity is attributed to their gene silencing capacity, by which miRNA binds $>100$ target mRNA sites with partial base complementarity, thus preventing de novo translation, and/or accelerating mRNA degradation. Collectively, this constitutes an important regulatory feature of the transcriptome [6]. miRNAs are co-expressed with their host genes [7], and thus influence downstream signaling events [8]. Dysregulated miRNA expression was associated with physiological and pathological processes, including cellular differentiation, angiogenesis, apoptosis, and embryogenesis $[9,10]$.

There is growing interest in the contribution of geneenvironmental interaction in RPL, and miRNA were proposed to constitute RPL at-risk factors [11, 12]. Given their diversity in regulating gene expression, the exact scope of action and influence of co-regulators and associated factors on miRNA activity remained poorly understood. Network modelling was proposed as key for deciphering miRNA activity. While this was described for number of pathological processes, miRNA network analysis in RPL pathogenesis was not previously reported.

The aims of the current study were to integrate the independent and different datasets in the analysis, thus overcoming the limitations caused by sample size and bias of statistical method. By summarizing large-scale gene expression data into limited modules, the study also aims to simplify data complexity, thus clarifying systematically the pathogenic mechanisms underlying RPL. Using direct association rationale, we provide for organization or connection information within module miRNA-genes. This may underscore the contribution of unannotated miRNA and genes to RPL pathogenesis.

\section{Methods}

\section{Data collection and preprocessing}

In the present study, the differentially expressed genes (DEGs) included genetically mutated genes, abnormally expressed protein genes, copy number variants, genes that alter DNA methylation, single nucleotide polymorphisms (SNPs), downregulated and upregulated genes. DEGs in the RPL were extracted from multiple databases (Additional file 1: Table S1). These databases include Online Mendelian Inheritance in Man (OMIM: catalog of human genes and genetic disorders that is freely available and updated daily) [13], GeneCards [14], Orphanet [15], Genetic Association Database (GAD, database of genetic association data from complex diseases and disorders) [16], and HuGE Navigator (provides a diseasecentered view of genetic association studies and information about genes explored in relation to a unique disease) [17]. We conducted a systematic review aimed at identifying genes with differential expression in RPL patients and tissues, using a combination of the key terms recurrent pregnancy loss, habitual abortion, idiopathic RPL, spontaneous recurrent miscarriage, associated polymorphisms, gene mutations, and gene expression profiling.

A list of genes associated with RPL included genes associated with cell adhesion (trophoblast/endometrium interaction), dysregulated immunity, coagulation and angiogenesis, and cell survival were also collected from literature search (Additional file 1: Table S1). P-match tool which is closely interconnected with the TRAN SFAC $^{\bullet}$ database, was utilized in identifying DNA transcription factor binding sites (TFBS) [18]. We focused only on TFs obtained using TransmiR. Prior to P-match, $1000 \mathrm{nt}$ promoter sequences of differentially expressed miRNAs were downloaded from Ensembl [19], using Regulatory Sequence Analysis Tools (RSAT) [20]. Pmatch matrix library comprises known TFBSs extracted from TRANSFAC [21], which allows searching of distinct TFBS. The "high quality vertebrate matrices only" option was selected as default, to reduce false-positive validation using $\mathrm{P}$-match.

\section{Differentially expressed miRNAs in RPL}

Differentially expressed miRNA (DEM) linked with RPL were extracted from miR2Disease [22], PhenomiR [23], and the Human microRNA Disease Database (HMDD) [24], using the following keywords: recurrent pregnancy loss, idiopathic RPL, spontaneous recurrent miscarriage AND microRNA (Additional file 1: Table S1). Additionally, we performed a secondary research based on systematic literature review of articles published between 2010 and 2017, and re-analyzed experimentally validated human miRNAs expression signatures in RPL from various biological sources (peripheral blood, and cell lines e.g., extravillous cytotrophoblast-derived cell line HTR8/SVneo (HTR8)) and information on aberrantly regulated miRNAs in patients with RPL as compared to healthy individuals. Our total search revealed 72 DEMs in RPL, as compared to healthy controls. Conflicting results have been found regarding expression levels of miRNAs. In order to increase the accuracy of our findings, only 37 of the most frequent DEM were collected (Table 1). 
Table 1 Differentially expressed microRNAs list

\begin{tabular}{|c|c|c|c|c|}
\hline Name & State & Sequence & Fold change & P-Value \\
\hline hsa-miR-146a-5p & UP & UGAGAACUGAAUUCCAUGGGUU & 4.45436783 & 0.0009 \\
\hline hsa-miR-125a-5p & UP & UCCCUGAGACCCUUUAACCUGUGA & 2.79 & $<0.001$ \\
\hline hsa-miR-155-5p & UP & UUAAUGCUAAUCGUGAUAGGGGUU & 3.96 & $<0.001$ \\
\hline hsa-miR-221-3p & UP & AGCUACAUUGUCUGCUGGGUUUC & 7.409 & 0.0070 \\
\hline hsa-miR-146b-5p & UP & UGAGAACUGAAUUCCAUAGGCUG & 5.108 & 0.0087 \\
\hline hsa-miR-320b & UP & AAAAGCUGGGUUGAGAGGGCAA & 2.637 & 0.0022 \\
\hline hsa-miR-133a-3p & UP & UUUGGUCCCCUUCAACCAGCUG & 0.009684 & $<0.001$ \\
\hline hsa-miR-101-3p & UP & UACAGUACUGUGAUAACUGAA & 3.614 & 0.0193 \\
\hline hsa-miR-223-3p & UP & UGUCAGUUUGUCAAAUACCCCA & 5.7 & $<0.001$ \\
\hline hsa-miR-92a-3p & UP & UAUUGCACUUGUCCCGGCCUGU & 2.662 & 0.0308 \\
\hline hsa-miR-148b-3p & UP & UCAGUGCAUCACAGAACUUUGU & 4.595 & 0.0434 \\
\hline hsa-miR-30d-5p & UP & UGUAAACAUCCCCGACUGGAAG & 4.361 & 0.0285 \\
\hline hsa-miR-23b-3p & UP & AUCACAUUGCCAGGGAUUACCAC & 3.430 & 0.0210 \\
\hline hsa-miR-423-3p & UP & AGCUCGGUCUGAGGCCCCUCAGU & 2.01 & 0.0332 \\
\hline hsa-miR-145-5p & UP & GUCCAGUUUUCCCAGGAAUCCCU & 0.722968 & 0.5116 \\
\hline hsa-miR-16-5p & UP & UAGCAGCACGUAAAUAUUGGCG & 0.879078 & 0.3559 \\
\hline hsa-miR-181a-5p & UP & AACAUUCAACGCUGUCGGUGAGU & 2.854288 & 0.0925 \\
\hline hsa-miR-424-5p & UP & CAGCAGCAAUUCAUGUUUUGAA & 0.127032 & 0.8565 \\
\hline hsa-miR-30d-5p & UP & UGUAAACAUCCCCGACUGGAAG & 0.604136 & 0.1879 \\
\hline hsa-miR-143-3p & UP & UGAGAUGAAGCACUGUAGCUC & 1.336424 & 0.2162 \\
\hline hsa-miR-27b-3p & UP & UUCACAGUGGCUAAGUUCUGC & 1.052276 & 0.4425 \\
\hline hsa-miR-17-5p & Down & CAAAGUGCUUACAGUGCAGGUAG & 0.35 & 0.0020 \\
\hline hsa-miR-19b-3p & Down & UGUGCAAAUCCAUGCAAAACUGA & 0.34 & $<0.001$ \\
\hline hsa-miR-559 & Down & UAAAGUAAAUAUGCACCAAAA & 0.390 & 0.0150 \\
\hline hsa-miR-365a-3p & Down & UAAUGCCCCUAAAAAUCCUUAU & 0.321 & 0.0318 \\
\hline hsa-miR-1182 & Down & GAGGGUCUUGGGAGGGAUGUGAC & 0.238 & 0.0186 \\
\hline hsa-miR-4284 & Down & GGGCUCACAUCACCCCAU & 0.428 & 0.0079 \\
\hline hsa-miR-4264 & Down & ACUCAGUCAUGGUCAUU & 0.113 & 0.0013 \\
\hline hsa-miR-4474-5p & Down & UUAGUCUCAUGAUCAGACACA & 0.196 & 0.0399 \\
\hline hsa-miR-22-5p & Down & AGUUCUUCAGUGGCAAGCUUUA & 0.337 & 0.0172 \\
\hline hsa-miR-372-5p & Down & CCUCAAAUGUGGAGCACUAUUCU & 0.22803727 & 0.0021 \\
\hline hsa-miR-451a & Down & AAACCGUUACCAUUACUGAGUU & -1.31401 & 0.0592 \\
\hline hsa-miR-21-5p & Down & UAGCUUAUCAGACUGAUGUUGA & -1.45161 & 0.4086 \\
\hline hsa-miR-149-5p & Down & UCUGGCUCCGUGUCUUCACUCCC & -1.530395 & 0.1853 \\
\hline hsa-miR-181b-5p & Down & AACAUUCAUUGCUGUCGGUGGGU & -2.329242 & 0.0434 \\
\hline
\end{tabular}

\section{Experimentally validated miRNA associations}

Experimentally validated human miRNAs and miRNAtarget interaction (MTI) datasets were extracted from miRtarbase and miRecords [25], and were validated by reporter assays, Western blotting, or microarrays with miRNA over-expression or knockdown [26]. Individual TFs were mapped to human TF list in ChIPBase, to distinguish among target genes [27]. Experimentally-confirmed TF-miRNAs regulations were extracted from TransmiR for assessing TF-miRNAs interplay [28]. This included information about miRNAs function, disease associations, and type of regulation (activation/repression). This association mapping between miRNAs and their host genes was done using NCBI, and miRBase, the latter containing information on 38,589 mRNA by March 2018 [29].

\section{Construction of differentially expressed and transcriptional networks}

A global network was constructed using CyTargetlinker [30], following extraction of regulatory associations 
between TFs, miRNAs, target genes and host genes. BridgeDb identifier mapping framework [31] was employed in standardizing miRNAs and genes designation, while Cytoscape was used in constructing differentially expressed networks (Additional file 1: Table S1). Lines between differentially-expressed nodal factors signify the interaction between different TFs, genes, and miRNAs in RPL; single nodes lacking regulatory miRNA association wereexcluded.

\section{Protein-protein interaction (PPI) network}

Protein-protein interaction (PPI) network, which identifies key elements in RPL based on interaction level, was acquired from STRING (Search Tool for Retrieval of Interacting Genes) database. DEG were mapped to STRING to evaluate PPI information, and visualized with Cytoscape. In addition, clusters of network highly intra-connected nodes were searched by MCODE (Molecular Complex Detection) Cytoscape network plug-in (Additional file 1: Table S1) [32].

\section{Functional enrichment analysis}

Gene ontology (GO) functional enrichment analysis was done using BiNGO (Biological Networks Gene Ontology) [33]. $P$ values were correct for false discovery rate (FDR) using Benjamini-Hochberg multiple testing correction. Kyoto Encyclopedia of Genes and Genomes (KEGG) pathway enrichment analysis of differentially expressed miRNA was performed by DNA Intelligent Analysis (DIANA)-miRPath v2.0 (Additional file 1: Table S1). Following DEMs inclusion miRNAs targets predicted with DIANA-microT-CDS and/or experimentally validated transcripts from TarBase v6.0 were selected; $P$ value for each pathway was obtained by Fisher's method [34]. The combinatorial effect of co-expressed miRNAs was evaluated by simultaneous analysis of multiple miRNAs; default settings were score cutoff of 0.8 for target prediction of 350 mRNA targets per miRNA, FDR to correct multiple hypothesis testing, and $P$-value of 0.05 .

\section{Results}

The occurrence and development of RPL are a complex process that involves genetic and epigenetic disorders. In the current study, a system-based networks' approach was used to examine the key regulatory associations between miRNAs, their target genes, their host genes, and TFs in RPL process. Experimentally validated data from databases and literature was collected and networks were constructed. Due to their complexity, data associated with this network does not provide for a clear description of the findings. Accordingly, we focused on analysis of differentially expressed network, RPL transcriptional network, and PPI network.
Differentially expressed miRNA and gene network of RPL A regulatory network for up- and down-regulated miRNAs and genes, was constructed for interpreting their differential expression in RPL at the transcriptional level (Fig. 1). This network, comprising 133 nodes/206 edges, consisted of 44 TFs, 36 target genes, 37 miRNAs, and associated host genes (Additional file 2: Table S2). Except for host genes, all other nodes were differentially expressed. The network nodes, directly or indirectly, affect other nodes. When combined, the interactions reveal the primary cause of RPL. The unaltered genes and miRNAs suggest that they are not related to RPL, they might not be actually protective. This suggests that RPL may be prevented by targeting these differentially expressed elements. miRNAs with high connectivity and module membership within a module were defined as "hub miRNAs", and were assumed to be functionally relevant. The top-10 hub miRNAs with the highest connectivity and module membership, were (in order): hsamiR-21-5p, hsa-miR-155-5p, hsa-miR-16-5p, hsa-miR17-5p, hsa-miR-146a-5p, hsa-miR-92a-3p, hsa-miR-1455p, hsa-miR-19b-3p, hsa-miR-221-3p and hsa-miR-1013p (Additional file 2: Table S2). These were selected based on the module sizes as hub miRNAs for further study.

As individual miRNA regulates gene expression, and multiple miRNAs modulate specific pathways, we explored the pathways regulated by hub miRNAs in RPL. Following inclusion of upregulated miRNAs, DIANAmiRPath identified 60 pathways as significantly enriched $(P<0.05)$. For simplicity and specificity, we focused only on pathways linked to RPL, the top 30 of which are shown in Table 2 (Additional file 2: Table S2). The most significantly enriched pathways regulated by hub miRNAs were TGF- $\beta$ signaling, cell cycle, adherens junction, fatty acid elongation, progesterone-mediated oocyte maturation, thyroid hormone signaling, and MAPK signaling pathways, which in turn target NF- $\mathrm{kB} 1, \mathrm{MYC}$ and E2F1 hub genes. Interestingly, the estrogen signaling pathway, which modulates reproductive functions, including progesterone production, utero placental blood flow, female secondary sexual characteristics, and maintenance of pregnancy, is also regulated by the selected hub miRNAs. Alteration in this pathway represents a cause of implantation failure and recurrent miscarriage. Significant enrichment was seen in 15 pathways influenced by hsa-miR-21-5p, hsa-miR-17-5p, and hsa-miR19b-3p (Table 2).

\section{Proposed transcriptional network of predicted TFs}

We predicted that DEM may contribute to targeting TF, since differentially expressed genes affect nucleic acid binding, and thus TF protein-binding activities. This allows assessment of the regulatory relationship between 


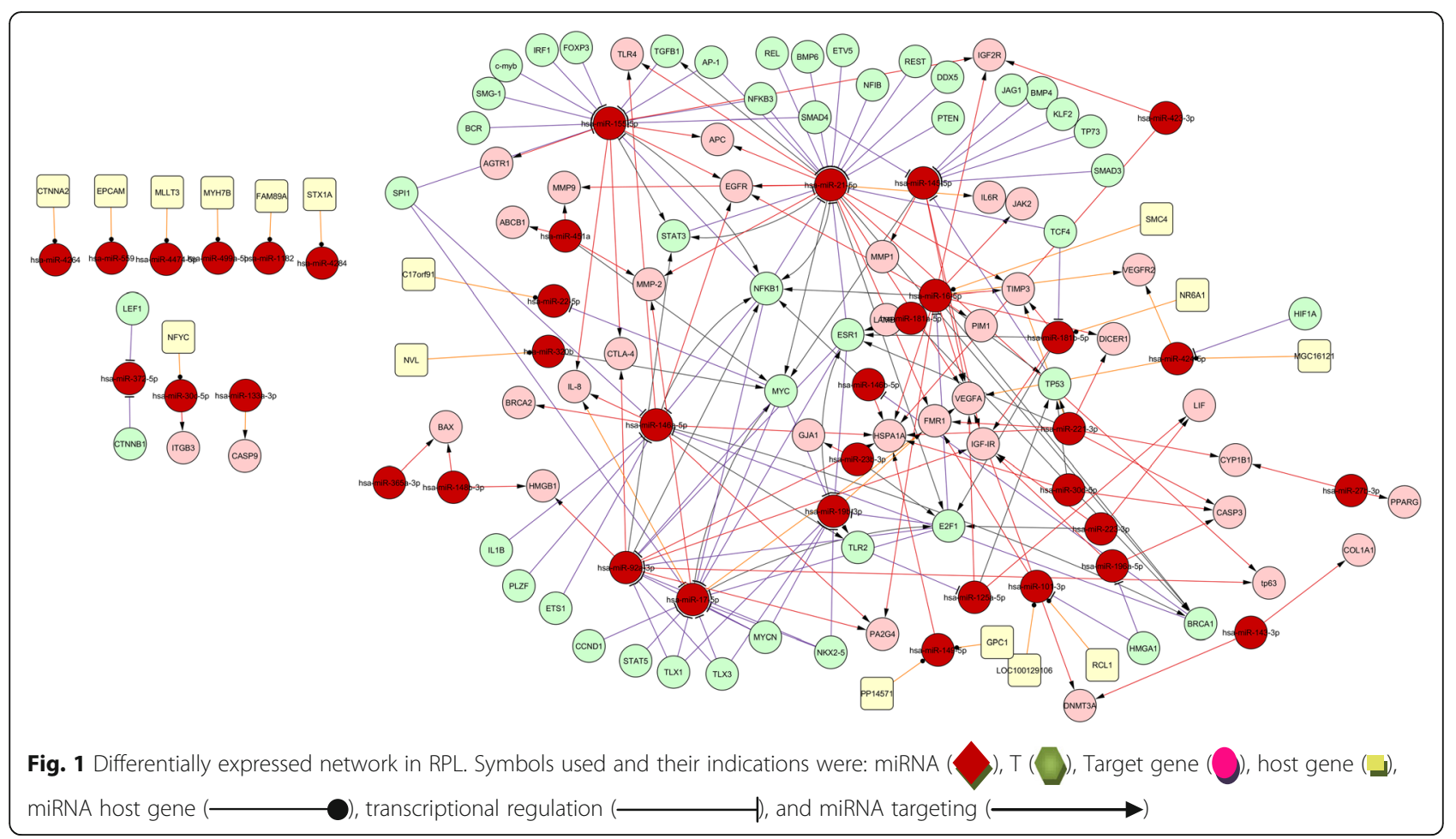

DEM and key TF genes. Analysis with predicted (Pmatch) and validated (transmiR) databases provided for construction of complex network, in which motifs between DEM and popular TFs were the building blocks (Fig. 2). A total of 44 TFs co-regulate 37 DEMs. Most proteins in this network are TFs that affect diverse cellular processes, such as angiogenesis, DNA damage response, development, morphogenesis, differentiation, and survival. Deregulated feedback in STAT3, NF-kB1, ESR1, MYC and E2F1 5 loops were also identified in this network.

Targeting NF-кB1 by hsa-miR-92a-3p, hsa-miR-165p, hsa-miR-155-5p, hsa-miR-146a-5p, hsa-miR-21-5p, and hsa-miR-146b-5p, regulating hsa-miR-21-5p, hsamiR-155-5p, hsa-miR-146a-5p, and hsa-miR-17-5p expression. NF- $\mathrm{B} 1$ is involved in two distinct apparently counter-regulatory feedback loops. The first involves NF-kB1 alone, and the second involving hsamiR-155-5p and hsa-miR-146a-5p. Heightened expression of hsa-miR-155-5p decreases $N F-\kappa B 1$ gene expression, resulting in reduced hsa-miR-155-5p expression. This increases $N F-\kappa B 1$ and subsequently hsa-miR-155-5p expression, and reactivates the loop. Similarly, feedback loop operates for $N F-\kappa B 1 /$ hsa-miR146a-5, which oscillates between increased and decreased states. This suggests that mixed regulation existing between hsa-miR-155-5p and hsa-miR-146a-5 with NF-kB1 involves multiple targets and pathways contributing to RPL. NF- $\mathrm{kB1}$ is also involved in a double negative feedback loop with hsa-miR-21-5p, allowing for "switch" type behavior, in which either NFKB1 or hsa-miR-21-5p, but not both, is expressed. The transition from one expression state to another state in this loop represents external modification of NF-kB1 or hsa-miR-21-5p expression.

On the other hand, hsa- miR-21-5p forms a separate feedback loop in targeting its regulators, NF-kB1 and STAT3. IntAct database and our PPI network confirm direct binding of NF- $\mathrm{kB} 1$ to several TFs, including STAT3, p53, ESR1, ATF3, SMAD3 and SMAD4. This suggests the existence of forward loop between ESR1, NF-кB1 and has-miR-21-5p, which precipitates a deregulated mechanism. This threegene pattern comprises an inducer (ESR1), which regulates the transcription of hsa-miR-21-5p, and both jointly regulating NF-KB1 activation. By targeting the same genes, the predicted network demonstrates that cross TF cooperation is needed for miRNA expression, highlighted by the regulation of hsa-miR-17-5p by STAT5, hsa-miR-21-5p by STAT3, and both hsa-miR-17-5p and hsa-miR-21-5p targeting MMP-2 expression.

Specific TF can cooperate with other TF in regulating the same miRNA, hence influencing the activity of the same target genes, exemplified by the regulation of hsamiR-17-5p by E2F1 and NFKB1, subsequently targets 
Table 2 Top 15 pathways significantly influenced by upregulated and downregulared miRNAs in RPL

\begin{tabular}{|c|c|c|c|}
\hline KEGG pathway & $\boldsymbol{p}$-value & Genes & miRNAs \\
\hline \multicolumn{4}{|l|}{ Upregulated miRNAs } \\
\hline TGF-beta signaling pathway & $3.89 \times 10^{-11}$ & 52 & 7 \\
\hline Adherens junction & $1.93 \times 10^{-10}$ & 51 & 7 \\
\hline Cell cycle & $5.00 \times 10^{-8}$ & 78 & 7 \\
\hline p53 signaling pathway & $4.11 \times 10^{-5}$ & 44 & 7 \\
\hline FoxO signaling pathway & $5.05 \times 10^{-5}$ & 73 & 7 \\
\hline Protein processing in endoplasmic reticulum & $5.05 \times 10^{-5}$ & 88 & 7 \\
\hline Fatty acid biosynthesis & $1.38 \times 10^{-4}$ & 4 & 4 \\
\hline Oocyte meiosis & $1.85 \times 10^{-4}$ & 58 & 7 \\
\hline Fatty acid metabolism & $3.93 \times 10^{-4}$ & 21 & 5 \\
\hline TNF signaling pathway & $7.64 \times 10^{-4}$ & 60 & 7 \\
\hline Estrogen signaling pathway & $6.65 \times 10^{-3}$ & 49 & 7 \\
\hline NOD-like receptor signaling pathway & 0.018 & 30 & 7 \\
\hline HIF-1 signaling pathway & 0.030 & 51 & 7 \\
\hline MAPK signaling pathway & 0.040 & 111 & 7 \\
\hline Circadian rhythm & 0.042 & 18 & 7 \\
\hline \multicolumn{4}{|l|}{ Downregulared miRNAs } \\
\hline Thyroid hormone signaling pathway & $9.24 \times 10^{-7}$ & 50 & 3 \\
\hline ECM-receptor interaction & $3.72 \times 10^{-6}$ & 24 & 3 \\
\hline Fatty acid elongation & $8.50 \times 10^{-5}$ & 9 & 3 \\
\hline Signaling pathways regulating pluripotency of stem cells & $1.24 \times 10^{-4}$ & 50 & 3 \\
\hline Prolactin signaling pathway & $1.96 \times 10^{-4}$ & 32 & 3 \\
\hline mRNA surveillance pathway & $3.98 \times 10^{-4}$ & 37 & 3 \\
\hline Lysine degradation & $7.17 \times 10^{-4}$ & 18 & 3 \\
\hline Insulin signaling pathway & $1.80 \times 10^{-3}$ & 52 & 3 \\
\hline Progesterone-mediated oocyte maturation & $2.40 \times 10^{-3}$ & 36 & 3 \\
\hline Apoptosis & $2.80 \times 10^{-3}$ & 32 & 3 \\
\hline Ubiquitin mediated proteolysis & $3.45 \times 10^{-3}$ & 52 & 3 \\
\hline PI3K-Akt signaling pathway & $4.35 \times 10^{-3}$ & 102 & 3 \\
\hline RNA degradation & $6.25 \times 10^{-3}$ & 31 & 3 \\
\hline RNA transport & 0.012 & 54 & 3 \\
\hline Endocytosis & 0.033 & 67 & 3 \\
\hline
\end{tabular}

MMP2, VEGFA, IL-8, and E2F1. These findings may be explained by temporary, but spatial interactions among mRNAs and miRNAs. TF, via miRNA, may indirectly influence the activity of other genes. This includes REL which regulates hsa-miR-21-5p, which targets STAT3, E2F1, NFKB1, MMP-2 and VEGFA. The TF-DEM network demonstrates that miRNA target TF sets, which physically interact with each other to silence the functional unit. The existence of a direct negative feedback loop between miRNA and TF highlights the limitation of functional units, as illustrated for NF-KB1, the limiting factor in the transcriptional regulation protein complex. It is noteworthy that miRNA and TF have similar regulatory nature, such as pleiotropy, regulation, and network motifs, which yield specific interactions that are propagated throughout the network.

While not abnormally expressed, a number of the network host genes and associated miRNAs appear to be involved in RPL. Accordingly, a single miRNA is depicted to be localized within multiple host genes, exemplified by the presence of hsa-miR-101-3p in RCL1 and within LOC100129106, and the localization of hsamiR-149-5p in GPC1 and PP14571. This provides for exploring more in depth the possible relationships between host genes and associated miRNA, especially when their miRNAs are differentially expressed. 

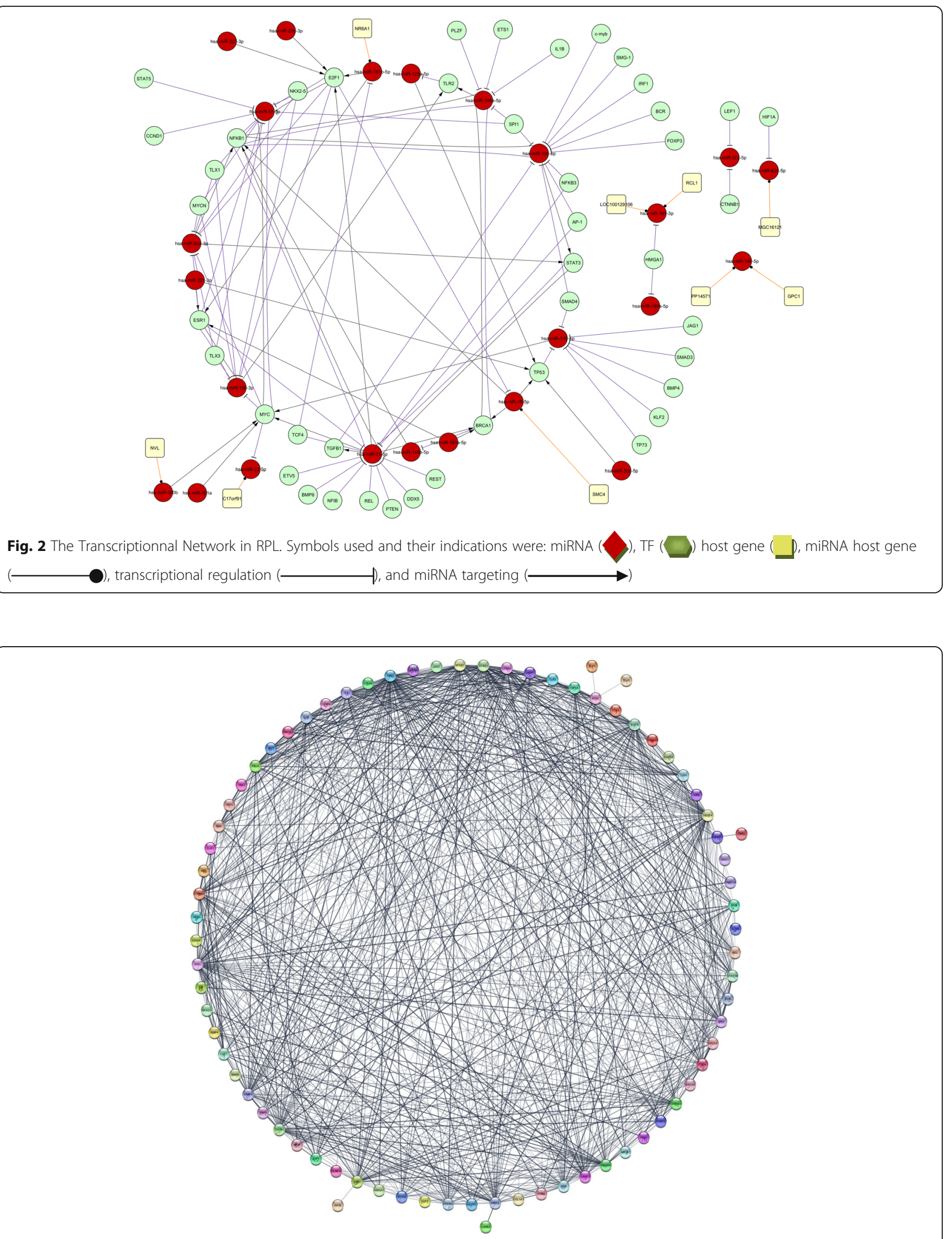

Fig. 3 RPL-PPI Network 


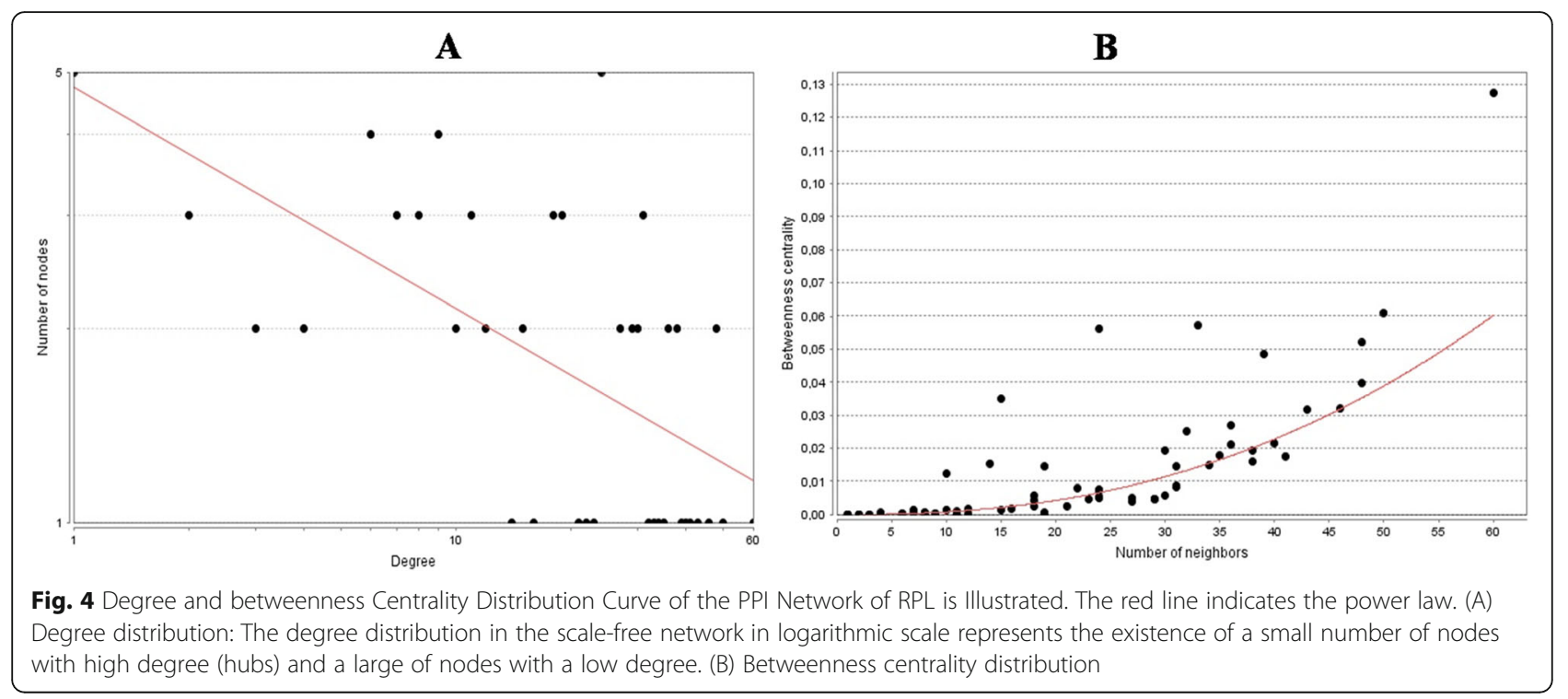

\section{Analysis of PPI network of the significantly RPL- associated proteins}

PPI networks of the significantly RPL-associated proteins, identified 77 nodes and 763 edges (Fig. 3). In addition, the power law of node degree distribution and centrality distribution were also performed (Fig. 4). Insofar as PPI networks are scale-free networks with power-law degree distribution, most nodes (proteins) have few connections to other node, whereas other nodes (specifically hubs) are connected to other nodes in the network. Common proteins (13 proteins), selected in hub and bottleneck states, and represented as hub-bottleneck proteins (Fig. 5), included EGFR, IL8, TP53, VEGFA, STAT3, TGFB1, CCND1, CTNNB1, CASP3, IL1B, MYC, ESR1 and NF-KB1.

Protein complexes (clusters) were identified by MCODE algorithm, and the global network was partitioned into three clusters, evaluated according to functionality (Table 3); function annotation performed using

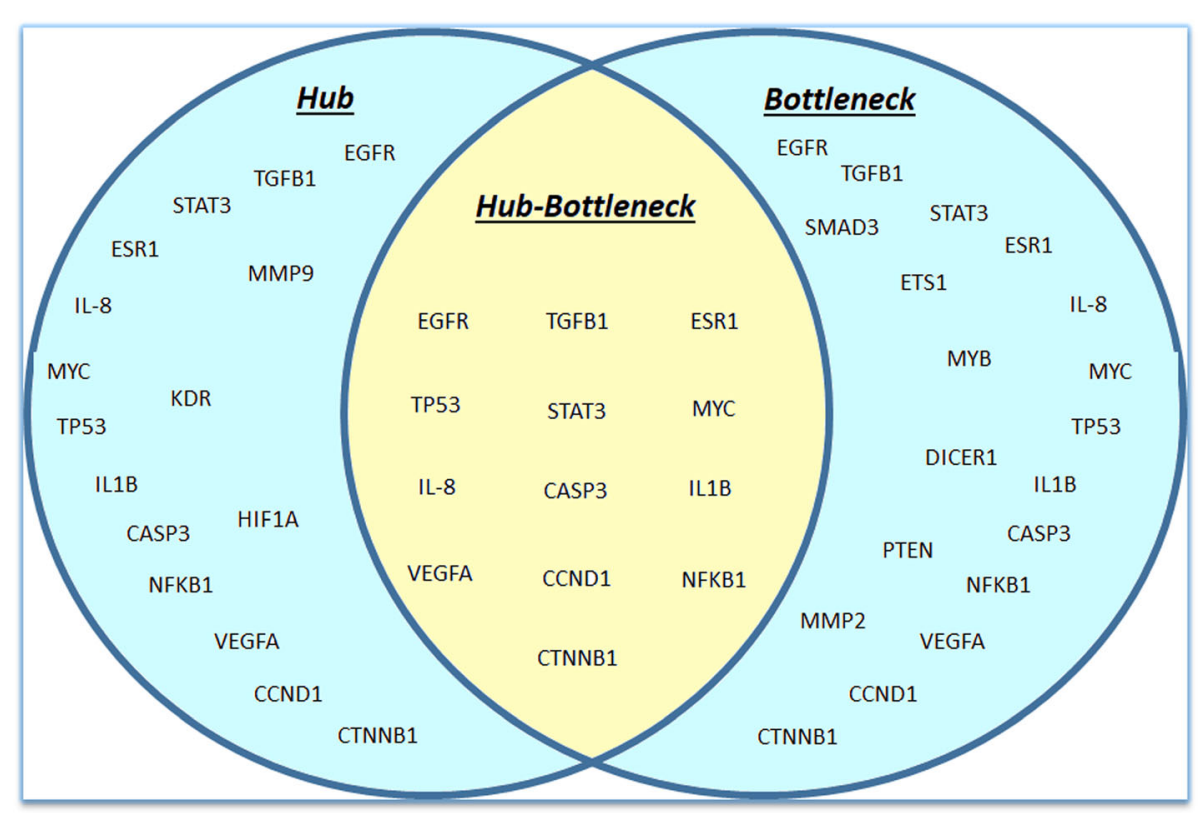

Fig. 5 The List of hub-bottleneck proteins. Common proteins (13 proteins), selected in hub and bottleneck states, and represented as hub-bottleneck proteins 
Table 3 Significant Clusters Related to the PPI Network of RPL and Their Properties

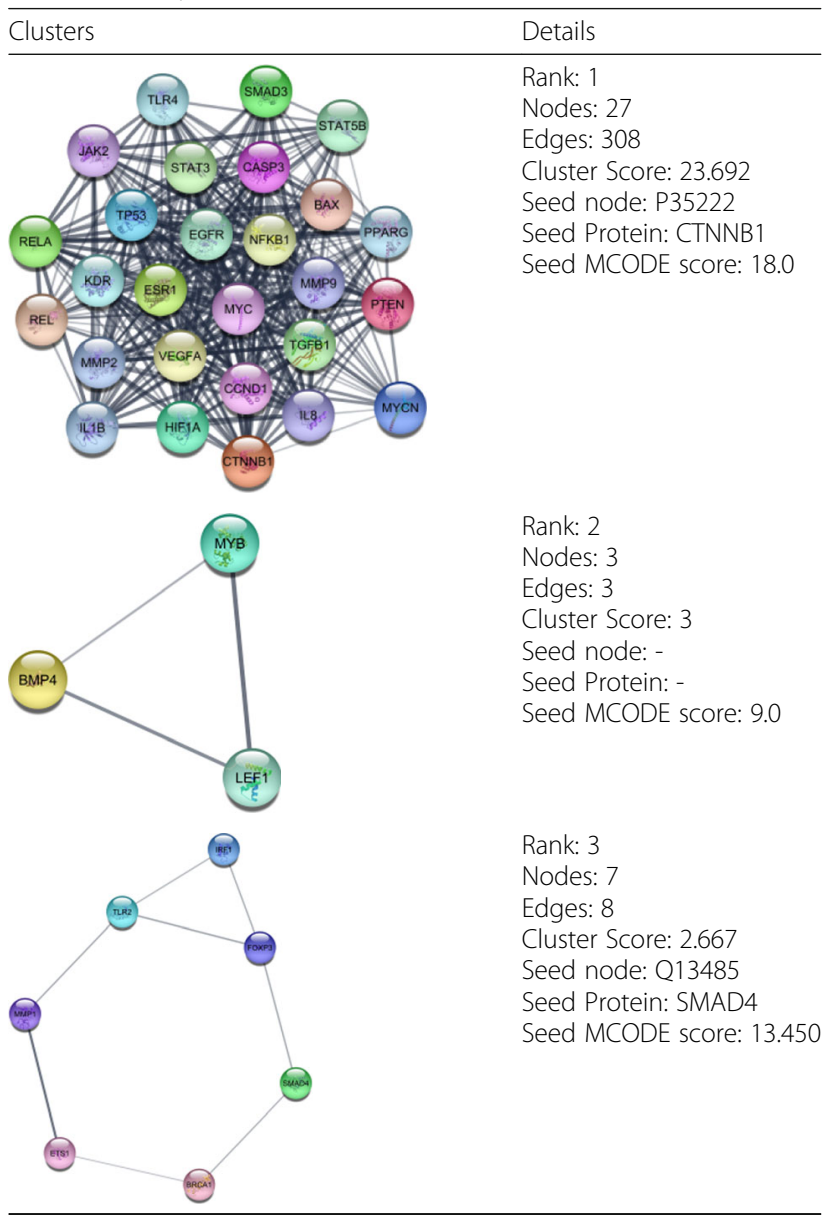

BiNGO plugin of Cytoscape (Table 4). Cluster 1 focused on regulation of cell proliferation, positive regulation of biological and developmental processes. Cluster 2 proteins were related to negative regulation of striated muscle tissue and muscle organ development. On the other hand, Cluster 3 members dealt with positive regulation of transcription from RNA polymerase II promoter, and DNA-dependent and positive regulation of RNA metabolic process.

\section{Discussion}

Bioinformatics analysis was utilized in identifying key miRNAs and genes to RPL. While earlier studies focused mostly on specific genes or metabolic pathways, our study included plethora of data generated to elucidate the role of interacting elements, and to understand their functional contribution in RPL. Our results identified select miRNA and TFs, and mapped their interaction and regulation for experimental validation in RPL. This study is the first to use bioinformatics in identifying and characterizing miRNA and gene expression profile in RPL. It is also the first to analyze the interaction among gene products, and to address the potential functionality of these genes in RPL.

The use of "omics" for identifying genes and effector mechanisms in RPL has challenges, mainly selection of study subjects (female patients, couple with miscarriages, fetus/placenta, and controls). The rely on genetic association studies focusing on candidate genes with pathological effect in RPL has limitations. While polymorphic variants of $\sim 100$ genes were investigated for possible association with RPL, results obtained were often inconclusive, and their diagnostic and prognostic utility remains questionable. Future directions in investigating biomolecular risk factors for RPL needs to integrate high-throughput profiling of genomes, transcriptomes, proteomes, metabolomes, and interactomes.

We identified 10 real hub miRNAs, and a further 80 mRNAs to be associated with RPL, and addressed their functionality through identification of miRNA-mRNA signaling pathways contributing to RPL pathogenesis. TGF- $\beta$ was the most significant pathway enriched by the upregulated miRNAs, namely hsa-miR-155-5p, hsa-miR16-5p, hsa-miR-92a-3, hsa-miR-145-5p, hsa-miR-221-3p, hsa-miR-101-3p, and hsa-miR-146a-5p. These miRNA target MYC gene, previously shown to be involved in trophoblast proliferation and apoptosis in RPL [35]. The anti-invasive action of TGF- $\beta$ on trophoblast is mediated by multiple mechanisms, including up-regulation of integrin expression, and reduction in migratory ability of invasive trophoblast [36]. TGF- $\beta$ also reduces matrix degradation via upregulating MMP tissue inhibitor expression.

Upregulated hsa-miR-16-5p targeted 31 genes in TGF- $\beta$ signaling, and was involved in $>5$ pathways, including adherens junction pathway. Differently expressed miRNAs affecting Wnt signaling, cell cycle, and adhesion molecules, were linked with defective embryo implantation in RPL [37]. Other pathways included MAPK pathway, which contributes to maintenance of normal pregnancy, and alteration in this pathway precipitates RPL [28]. MAPK pathway was influenced by hsa-miR-16-5p and p53 pathways, and modulated by hsa-miR-16-5p and hsa-miR-155-5p. In addition, altered p53 signaling pathway, involved in apoptosis and cell cycle regulation [38], results in enhanced placental apoptosis, and RPL through upregulation of hsa-miR-155-5p [39]. As overexpression of hsa-miR-155-5p enhances, while its antagonism impairs NK cell-mediated cytotoxic activity, this suggesting targeting hsa-miR-155 in managing RPL [40]. Similarly, 15 pathways affected by downregulated miRNAs were noted, including thyroid hormone signaling and prolactin signaling pathways. Altered thyroid hormone level can lead to abnormal sexual 
Table 4 Functional annotations of the protein complexes (clusters)

\begin{tabular}{|c|c|c|c|}
\hline GO-ID & corr $p$-value & $x$ & Description \\
\hline \multicolumn{4}{|c|}{ Module 1} \\
\hline 42,127 & 4.3349E-18 & 20 & regulation of cell proliferation \\
\hline 48,518 & 6.0837E-18 & 25 & positive regulation of biological process \\
\hline 50,793 & $1.8202 \mathrm{E}-17$ & 19 & regulation of developmental process \\
\hline 48,522 & $1.8202 \mathrm{E}-17$ & 24 & positive regulation of cellular process \\
\hline 42,221 & 2.9470E-17 & 22 & response to chemical stimulus \\
\hline 51,239 & $6.8692 \mathrm{E}-17$ & 20 & regulation of multicellular organismal process \\
\hline 9893 & $9.7918 \mathrm{E}-16$ & 19 & positive regulation of metabolic process \\
\hline 51,173 & $1.0140 \mathrm{E}-15$ & 17 & positive regulation of nitrogen compound metabolic process \\
\hline 42,981 & $1.1601 \mathrm{E}-15$ & 18 & regulation of apoptosis \\
\hline 43,067 & $1.2325 \mathrm{E}-15$ & 18 & regulation of programmed cell death \\
\hline 10,941 & $1.2938 \mathrm{E}-15$ & 18 & regulation of cell death \\
\hline 31,328 & $1.9038 \mathrm{E}-15$ & 17 & positive regulation of cellular biosynthetic process \\
\hline 9891 & $2.2668 \mathrm{E}-15$ & 17 & positive regulation of biosynthetic process \\
\hline 10,628 & $3.7320 \mathrm{E}-15$ & 16 & positive regulation of gene expression \\
\hline 10,604 & 4.0493E-15 & 18 & positive regulation of macromolecule metabolic process \\
\hline \multicolumn{4}{|c|}{ Module 2} \\
\hline 45,843 & $2.1117 \mathrm{E}-4$ & 2 & negative regulation of striated muscle tissue development \\
\hline 48,635 & $2.1117 \mathrm{E}-4$ & 2 & negative regulation of muscle organ development \\
\hline \multicolumn{4}{|c|}{ Module 3} \\
\hline 45,944 & $5.4874 \mathrm{E}-7$ & 6 & positive regulation of transcription from RNA polymerase II promoter \\
\hline 45,893 & $9.0278 \mathrm{E}-7$ & 6 & positive regulation of transcription, DNA-dependent \\
\hline 51,254 & $9.0278 \mathrm{E}-7$ & 6 & positive regulation of RNA metabolic process \\
\hline 45,941 & $1.4582 \mathrm{E}-6$ & 6 & positive regulation of transcription \\
\hline 10,628 & $1.5514 \mathrm{E}-6$ & 6 & positive regulation of gene expression \\
\hline 45,935 & $1.9401 \mathrm{E}-6$ & 6 & positive regulation of nucleobase, nucleoside, nucleotide and nucleic acid metabolic process \\
\hline 10,557 & $1.9401 \mathrm{E}-6$ & 6 & positive regulation of macromolecule biosynthetic process \\
\hline 51,173 & $1.9401 \mathrm{E}-6$ & 6 & positive regulation of nitrogen compound metabolic process \\
\hline 31,328 & $2.3309 \mathrm{E}-6$ & 6 & positive regulation of cellular biosynthetic process \\
\hline 9891 & $2.3309 \mathrm{E}-6$ & 6 & positive regulation of biosynthetic process \\
\hline 10,552 & $2.3309 E-6$ & 4 & positive regulation of gene-specific transcription from RNA polymerase II promoter \\
\hline 6357 & $2.3309 E-6$ & 6 & regulation of transcription from RNA polymerase II promoter \\
\hline 43,193 & $6.8613 \mathrm{E}-6$ & 4 & positive regulation of gene-specific transcription \\
\hline 10,604 & $7.9390 \mathrm{E}-6$ & 6 & positive regulation of macromolecule metabolic process \\
\hline 31,325 & $8.6648 \mathrm{E}-6$ & 6 & positive regulation of cellular metabolic process \\
\hline
\end{tabular}

development, menstruation irregularity, and likely RPL, while hyperprolactinemia affects hypothalamicpituitary-ovarian axis, resulting in a shorter luteal phase [41].

Regulation of transcriptional and post-transcriptional events were obtained by constructing curated miRNATF regulatory network, the smaller significant subnetwork modules centering on NF-kB1 gene was key. Direct TF-miRNA feedback loops identified two NF- $\mathrm{kB} 1$ loops involving hsa-miR-155-5p and hsa-miR-146a-5p, and a third involving NF-kB1 and hsa-miR-21-5p. NF$\kappa B 1$ regulates innate and adaptive immunity, and induces inflammation by stimulating the expression of pro-inflammatory genes, especially IL-6 and IL-8 [42]. Similarly, hsa-miR-146a-5p negatively regulates the expression of IL-6 and IL-8 [43]. Moreover, NF-kB1 activity is regulated by the ubiquitin-protein ligase pellino homolog 1 , the expression of which is negatively regulated by hsa-miR-21-5p and thus is inhibitory of NF- $\mathrm{kB} 1$ [44]. On the other hand, hsa-miR-21-5p was shown to be activated by IL- 6 in a STAT3-dependent manner [45]. A strong link between miR-146a-5p and hsa-miR21-5p, and with NF- $\kappa B 1$ was seen, predicting that NF$\kappa B 1$ interaction with hsa-miR-146a-5p affects IL-8 expression, suggesting feedback loops involving NF-kB1 and hsa-miR-146a-5p or hsa-miR-21-5p for controlling 
RPL-associated inflammation. Earlier studies documented negative feedback comprising hsa-miR-21-5p with NF-kB1, which was linked with altered NF-kB1 and IL-6 activity, and additional negative feedback loop provided by hsa-miR-146 [46]. Accordingly, differences in the strength of the two loops provides for the needed signals for RPL development.

By providing target-specific post-transcriptional repression mechanisms, miRNA-TF network underscores the complexity of TF-miRNA motifs. Compared to TF, miRNA can quickly terminate, or alternatively resume protein translation by binding to, or disassociating from an already transcribed mRNA. This provides for rapid and adaptive changes in gene expression. Although they are subject to differential expression, the exact role of miRNA in RPL remains to be seen. The proposed transcriptional network could explain this involvement. For example, while NF-kB1-hsa-miR-146a-5p loops underscore miRNA effect in sustaining normal immune homeostasis, abnormal hsa-miR-146a-5p expression can drive inflammation; a hypothesis that could not have been made otherwise.

PPI network analysis allows for discrimination of key nodes, and is useful as network biomarker discovery in RPL. Based on their significance in the network, PPI network of 77 RPL-associated proteins were constructed as predictors of drug targets. Crucial nodes which interacted with and controlled the expression of other network nodes, were introduced as essential in RPL PPI network. Localized in Cluster 1, 13 key nodes were found to be related to RPL, individually or in combination, of which CTNNB1 appears the most significant. Mothers against decapentaplegic homolog 4 (SMAD4) protein was also found to be a crucial node in the PPI network, and was also found as a seed protein in Cluster 3 . SMAD4, previously reported as key component in TGF- $\beta$ signaling [47], are intracellular transducers of TGF- $\beta$ superfamily, and PSG9/SMAD4 complex recruite cytoplasmic SMAD2/3 forming a complex, which enhanced SMAD4 nuclear retention [48]. PSG9-SMAD4 complex was shown to activate the expression of angiogenesis-related genes, including VEGFA, thus contributing to the endometrial angiogenesis affecting both conception and fetal development [49].

STAT3 is a crucial hub-bottleneck protein involved in cytokine and growth factor signaling, and is a key regulator of anti-inflammatory signaling pathway. Altered STAT activity was implicated in adverse pregnancy outcomes, and a 3'-UTR STAT3 variant contributes to RPL by precipitating a local inflammatory state [50]. Since endometrium signaling involves STAT pathway [50], defective STAT signaling due to attenuated endometrial STAT3-associated tyrosine kinase activity [51, 52], or altered availability of cytokine/growth factor-driven receptor engagement was seen in RPL, suggesting a role in unexplained infertility. CTNNB1, identified as the seed node in Cluster 1, is present in 21 KEGG pathways, including Wnt signaling pathway, suggest that altered CTNNB1 expression contributed to RPL pathogenesis. With regards to apoptosis-related genes and pathways, several evidences confirmed apoptosis of trophoblast cells in early pregnancy [53]. Implantation and growth of blastocyst, regression and reconstruction of decidual tissues, remodeling of placental structure and other processes are closely related to apoptosis, were also present [53]. A balance between apoptosis and proliferation of villous and decidual cells during pregnancy and adverse pregnancy outcome, including RPL, are commonly associated with the excessive apoptotic cells.

\section{Conclusions}

Using bioinformatics, results of the present study provides for theoretical framework toward personalized RPL therapy. Hub-bottleneck proteins, had critical role and high importance in physiopathology of RPL, can serve a diagnostic, even prognostic, role in RPL pathobiology. It is tempting to speculate that several of the identified miRNAs and proteins can potentially serve as diagnostic or therapeutic agents. To this end, prioritization of these targets, and validation of the results using direct cellular analysis, and where possible examination of the impact of the blockade of their expression and activity will be needed for further examination. We favor the notion that drugs designed against these proteins can be important in controlling and managing of RPL.

\section{Supplementary information}

Supplementary information accompanies this paper at https://doi.org/10. 1186/s12920-020-00730-z.

Additional file 1: Table S1. Data banks/repositories corresponding to datasets analyzed in this study.

Additional file 2: Table S2. Accession Number of Identified miRNA.

\begin{abstract}
Abbreviations
BiNGO: Biological Networks Gene Ontology; DEGs: Differentially expressed genes; DEMs: Differentially expressed miRNAs; ESAS: Exome sequencing analysis studies; FDR: False discovery rate; GAD: Genetic Association Database; GWAS: Genome wide association studies; GO: Gene ontology; KEGG: Kyoto Encyclopedia of Genes and Genomes; MCODE: Molecular Complex Detection; miRNAs: microRNAs; MTI: miRNA-target interaction; OMIM: Online Mendelian Inheritance in Man; PPI: Protein-protein interaction; RPL: Recurrent pregnancy loss; STRING: Search Tool for Retrieval of Interacting Genes; TF: Transcription factor; TFBS: Transcription factor binding sites
\end{abstract}

\section{Acknowledgments}

The authors wish to thank Dr. Ikram Sghaier for her helpful suggestions while drafting the manuscript.

Authors' contributions

WB: Drafted the manuscript, IS: Bioinformatics analysis and data mining, AA: Statistical analysis, AH: Literature review and drafting of Discussion, SF: 
Literature review, SM: Bioinformatics and statistical analysis, WA: Edited the manuscript, corresponding author. The author(s) read and approved the final manuscript.

\section{Funding}

The study did not receive funding from institutional or external sources.

\section{Availability of data and materials}

Three types of specific datasets were used. First, experimentally validated datasets of human miRNAs and target genes were collected from miRtarbase (http://mirtarbase.mbc.nctu.edu.tw) available at (http://mirtarbase. mbc.nctu.edu.tw/cache/download/7.0/hsa_MTI.xlsx). The second dataset consisting of targeting associations of human miRNAs and genes was collected from miRecords (http://c1.accurascience.com/miRecords/), which is available at (http://c1.accurascience.com/miRecords/download_data.php?v= 4). Lastly, experimentally confirmed TF-miRNA regulations in TransmiR (http:// www.cuilab.cn/transmir) was used for analyzing the interplay between TFs and miRNAs, and is available at (http://www.cuilab.cn/files/images/transmir2/ download/literature/hsa.xlsx). The names and direct links of the data banks/ repositories corresponding to the datasets obtained from web-based sources and subsequently analysed in the study are found in Suplementary Table 1: Data banks/repositories corresponding to datasets analyzed in this study. The accession number and direct web links of the identified miRNA appear in Additional file 2: Table S2.

\section{Ethics approval and consent to participate}

None required, given the scope of the manuscript.

\section{Consent for publication}

Not applicable.

\section{Competing interests}

The authors declare that they have no conflict of interest to disclose.

\section{Author details}

${ }^{1}$ Research Unit of Clinical and Molecular Biology, Department of Biochemistry, Faculty of Pharmacy of Monastir, University of Monastir, Monastir, Tunisia. ${ }^{2}$ Faculty of Science of Bizerte, University of Carthage, Bizerte, Tunisia. ${ }^{3}$ Molecular and Cellular Hematology Laboratory, Institut Pasteur de Tunis, Université Tunis El Manar, Tunis, Tunisia. ${ }^{4}$ Laboratory of Physiology, Faculty of Medicine of Tunis, la Rabta, 1007 Tunis, Tunisia. ${ }^{5}$ Department of Obstetrics and Gynecology, Fattouma Bourguiba University Hospital, Monastir, Tunisia. ${ }^{6}$ Faculty of Sciences, El Manar University, Tunis, Tunisia. ${ }^{7}$ College of Health Sciences, Abu Dhabi University, Abu Dhabi, United Arab Emirates.

Received: 15 October 2019 Accepted: 12 May 2020

Published online: 01 June 2020

\section{References}

1. American College of O, Gynecologists. ACOG practice bulletin. Management of recurrent pregnancy loss. Number 24, February 2001. (replaces technical bulletin number 212, September 1995). American College of Obstetricians and Gynecologists. Int J Gynaecol Obstet. 2002;78:179-90.

2. Bahia W, Finan RR, Al-Mutawa M, Haddad A, Soua A, Janhani F, et al. Genetic variation in the progesterone receptor gene and susceptibility to recurrent pregnancy loss: a case-control study. BJOG. 2018;125:761-2.

3. Puscheck EE, Jeyendran RS. The impact of male factor on recurrent pregnancy loss. Curr Opin Obstet Gynecol. 2007;19:222-8.

4. Ranganathan K, Sivasankar V. MicroRNAs - biology and clinical applications. J Oral Maxillofac Pathol. 2014;18:229-34

5. Catalanotto C, Cogoni C, Zardo G. MicroRNA in control of gene expression: an overview of nuclear functions. International journal of molecular sciences. Int J Mol Sci. 2016;17:E1712.

6. Hannafon BN, Ding WQ. Functional role of microRNAs in the progression of breast ductal carcinoma in situ. Am J Pathol. 2019;189:966-74.

7. Baskerville S, Bartel DP. Microarray profiling of microRNAs reveals frequent coexpression with neighboring miRNAs and host genes. Rna. 2005;11:241-7.

8. Cao G, Huang B, Liu Z, Zhang J, Xu H, Xia W, et al. Intronic miR-301 feedback regulates its host gene, ska2, in A549 cells by targeting MEOX2 to affect ERK/CREB pathways. Biochem Biophys Res Commun. 2010;396:978-82.
9. Jairajpuri DS, Almawi WY. MicroRNA expression pattern in pre-eclampsia (review). Mol Med Rep. 2016;13:2351-8.

10. Soltani I, Gharbi H, Hassine IB, Bouguerra G, Douzi K, Teber M, et al. Regulatory network analysis of microRNAs and genes in imatinib-resistant chronic myeloid leukemia. Funct Integr Genomics. 2017;17:263-77.

11. Hu Y, Liu CM, Qi L, He TZ, Shi-Guo L, Hao CJ, et al. Two common SNPs in pri-miR-125a alter the mature miRNA expression and associate with recurrent pregnancy loss in a Han-Chinese population. RNA Biol. 2011;8: $861-72$.

12. Jeon YJ, Choi YS, Rah H, Kim SY, Choi DH, Cha SH, et al. Association study of microRNA polymorphisms with risk of idiopathic recurrent spontaneous abortion in Korean women. Gene. 2012;494:168-73.

13. Hamosh A, Scott AF, Amberger J, Bocchini C, Valle D, McKusick VA. Online Mendelian inheritance in man (OMIM), a knowledgebase of human genes and genetic disorders. Nucleic Acids Res. 2002;30:52-5.

14. Safran M, Dalah I, Alexander J, Rosen N, Iny Stein T, Shmoish M, et al. GeneCards version 3: the human gene integrator. Database (Oxford). 2010; 2010:baq020.

15. Weinreich SS, Mangon R, Sikkens JJ, Teeuw ME, Cornel MC. Orphanet: a European database for rare diseases. Ned Tijdschr Geneeskd. 2008;152: 518-9.

16. Becker KG, Barnes KC, Bright TJ, Wang SA. The genetic association database. Nat Genet. 2004;36:431-2.

17. Yu W, Clyne M, Khoury MJ, Gwinn M. Phenopedia and Genopedia: diseasecentered and gene-centered views of the evolving knowledge of human genetic associations. Bioinformatics. 2010;26:145-6.

18. Chekmenev DS, Haid C, Kel AE. P-match: transcription factor binding site search by combining patterns and weight matrices. Nucleic Acids Res. 2005; 33:W432-7.

19. Cunningham F, Amode MR, Barrell D, Beal K, Billis K, Brent $S$, et al. Ensembl 2015. Nucleic Acids Res. 2015;43(Database issue):D662-9.

20. Nguyen NTT, Contreras-Moreira B, Castro-Mondragon JA, Santana-Garcia W, Ossio R, Robles-Espinoza CD, et al. RSAT 2018: regulatory sequence analysis tools 20th anniversary. Nucleic Acids Res. 2018;46(W1):W209-14.

21. Wingender E, Dietze $P$, Karas H, Knuppel R. TRANSFAC: a database on transcription factors and their DNA binding sites. Nucleic Acids Res. 1996;24: 238-41.

22. Jiang $Q$, Wang $Y$, Hao $Y$, Juan $L$, Teng $M$, Zhang $X$, et al. miR2Disease: a manually curated database for microRNA deregulation in human disease. Nucleic Acids Res. 2009:37(Database issue):D98-104.

23. Ruepp A, Kowarsch A, Theis F. PhenomiR: microRNAs in human diseases and biological processes. Methods Mol Biol. 2012;822:249-60.

24. Lu M, Zhang Q, Deng M, Miao J, Guo Y, Gao W, et al. An analysis of human microRNA and disease associations. PLoS One. 2008;3:e3420.

25. Xiao F, Zuo Z, Cai G, Kang S, Gao X, Li T. miRecords: an integrated resource for microRNA-target interactions. Nucleic Acids Res. 2009;37(Database issue): D105-10.

26. Hsu SD, Lin FM, Wu WY, Liang C, Huang WC, Chan WL, et al. miRTarBase: a database curates experimentally validated microRNA-target interactions. Nucleic Acids Res. 2011;39(Database issue):D163-9.

27. Zhou KR, Liu S, Sun WJ, Zheng LL, Zhou H, Yang JH, et al. ChIPBase v2.0: decoding transcriptional regulatory networks of non-coding RNAs and proteincoding genes from ChIP-seq data. Nucleic Acids Res. 2017;45(D1):D43-50.

28. Wang J, Lu M, Qiu C, Cui Q. TransmiR: a transcription factor-microRNA regulation database. Nucleic Acids Res. 2010;38(Database issue):D119-22.

29. Kozomara A, Birgaoanu M, Griffiths-Jones S. miRBase: from microRNA sequences to function. Nucleic Acids Res. 2019;47(D1):D155-62.

30. Kutmon M, Kelder T, Mandaviya P, Evelo CT, Coort SL. CyTargetLinker: a cytoscape app to integrate regulatory interactions in network analysis. PLoS One. 2013;8: e82160.

31. van lersel MP, Pico AR, Kelder T, Gao J, Ho I, Hanspers K, et al. The BridgeDb framework: standardized access to gene, protein and metabolite identifier mapping services. BMC Bioinformatics. 2010;11:5.

32. Bader GD, Hogue CW. An automated method for finding molecular complexes in large protein interaction networks. BMC Bioinformatics. 2003;4:2.

33. Maere S, Heymans K, Kuiper M. BiNGO: a Cytoscape plugin to assess overrepresentation of gene ontology categories in biological networks. Bioinformatics. 2005;21(16):3448-9.

34. Vlachos IS, Kostoulas N, Vergoulis T, Georgakilas G, Reczko M, Maragkakis M, et al. DIANA miRPath v.2.0: investigating the combinatorial effect of microRNAs in pathways. Nucleic Acids Res. 2012;40:W498-504. 
35. Wu F, Tian F, Zeng W, Liu X, Fan J, Lin Y, et al. Role of peroxiredoxin2 downregulation in recurrent miscarriage through regulation of trophoblast proliferation and apoptosis. Cell Death Dis. 2017;8:e2908.

36. Cheng JC, Chang HM, Leung PC. Transforming growth factor-beta1 inhibits trophoblast cell invasion by inducing snail-mediated down-regulation of vascular endothelial-cadherin protein. J Biol Chem. 2013;288:33181-92.

37. Revel A, Achache H, Stevens J, Smith Y, Reich R. MicroRNAs are associated with human embryo implantation defects. Hum Reprod. 2011;26:2830-40.

38. Chen J. The cell-cycle arrest and apoptotic functions of p53 in tumor initiation and progression. Cold Spring Harb Perspect Med. 2016;6:a026104.

39. Shang W, Shu MM, Liu M, Wang AM, Lv LB, Zhao Y, et al. Elevated expressions of p53, CDKNA1, and Bax in placental villi from patients with recurrent spontaneous abortion. Eur Rev Med Pharmacol Sci. 2013;17:3376-80.

40. Liu X, Wang Y, Sun Q, Yan J, Huang J, Zhu S, et al. Identification of microRNA transcriptome involved in human natural killer cell activation. Immunol Lett. 2012;143:208-17.

41. Schliep KC, Mumford SL, Hammoud AO, Stanford JB, Kissell KA, Sjaarda LA, et al. Luteal phase deficiency in regularly menstruating women: prevalence and overlap in identification based on clinical and biochemical diagnostic criteria. J Clin Endocrinol Metab. 2014;99:E1007-14.

42. Pitman H, Innes BA, Robson SC, Bulmer JN, Lash GE. Altered expression of interleukin-6, interleukin-8 and their receptors in decidua of women with sporadic miscarriage. Hum Reprod. 2013;28:2075-86.

43. Pfeiffer D, Rossmanith E, Lang I, Falkenhagen D. miR-146a, miR-146b, and miR-155 increase expression of IL-6 and IL-8 and support HSP10 in an in vitro sepsis model. PLoS One. 2017:12:e0179850.

44. Marquez RT, Wendlandt E, Galle CS, Keck K, McCaffrey AP. MicroRNA-21 is upregulated during the proliferative phase of liver regeneration, targets Pellino-1, and inhibits NF-kappaB signaling. Am J Physiol Gastrointest Liver Physiol. 2010;298:G535-41.

45. Niopoulos D, Jaeger SA, Hirsch HA, Bulyk ML, Struhl K. STAT3 activation of miR-21 and miR-181b-1 via PTEN and CYLD are part of the epigenetic switch linking inflammation to cancer. Mol Cell. 2010;39:493-506.

46. Xue X, Xia W, Wenzhong H. A modeled dynamic regulatory network of NFkappaB and IL-6 mediated by miRNA. Biosystems. 2013;114:214-8.

47. Macias MJ, Martin-Malpartida P, Massague J. Structural determinants of Smad function in TGF-beta signaling. Trends Biochem Sci. 2015;40:296-308.

48. Zhao M, Mishra L, Deng CX. The role of TGF-beta/SMAD4 signaling in cancer. Int J Biol Sci. 2018;14:111-23.

49. Yang L, Hu S, Tan J, Zhang X, Yuan W, Wang Q, et al. Pregnancy-specific glycoprotein 9 (PSG9), a driver for colorectal cancer, enhances angiogenesis via activation of SMAD4. Oncotarget. 2016;7:61562-74.

50. Finan RR, Mustafa FE, Al-Zaman I, Madan S, Issa AA, Almawi WY. STAT3 polymorphisms linked with idiopathic recurrent miscarriages. Am J Reprod Immunol. 2010;63:22-7.

51. Catalano RD, Johnson MH, Campbell EA, Charnock-Jones DS, Smith SK, Sharkey AM. Inhibition of Stat3 activation in the endometrium prevents implantation: a nonsteroidal approach to contraception. Proc Natl Acad Sci U S A. 2005;102:8585-90

52. Chen CL, Hsieh FC, Lieblein JC, Brown J, Chan C, Wallace JA, et al. Stat3 activation in human endometrial and cervical cancers. Br J Cancer. 2007;96: 591-9.

53. Su RW, Fazleabas AT. Implantation and establishment of pregnancy in human and nonhuman primates. Adv Anat Embryol Cell Biol. 2015;216:189213.

\section{Publisher's Note}

Springer Nature remains neutral with regard to jurisdictional claims in published maps and institutional affiliations.

Ready to submit your research? Choose BMC and benefit from:

- fast, convenient online submission

- thorough peer review by experienced researchers in your field

- rapid publication on acceptance

- support for research data, including large and complex data types

- gold Open Access which fosters wider collaboration and increased citations

- maximum visibility for your research: over $100 \mathrm{M}$ website views per year

At $\mathrm{BMC}$, research is always in progress.

Learn more biomedcentral.com/submissions 